\title{
Sur les interrogatives indirectes construites avec sur: leur distribution avec verbes à deux compléments
}

\author{
Takuya Nakamura \\ Institut Gaspard Monge (IGM) \\ nakamura@univ-mlv.fr
}

\section{Introduction}

Dans cette étude, nous nous intéressons à la distribution des interrogatives indirectes (I.I.) introduites par une préposition. Comparées à l'abondance dans la littérature des études sur les I.I. en général, les descriptions de relations syntagmatiques entre prépositions et I.I. ne sont pas abondantes, probablement à cause du style oral qui les stigmatisent. Ces phénomènes ont été peu décrits, certes, mais ils existent bel et bien dans le français qu'on rencontre tous les jours :

Quand à conclure à qui a le monopole de quoi, vous vous appuyez sur bien peu de choses pour tirer vos conclusions... (Internet)

Chaque concert est le dernier. Il dépend de qui l'écoute ${ }^{1}$ (Le Monde, 1994)

Il est totalement inacceptable qu'un fonctionnaire international critique les Etats-Unis (...) ou d'ailleurs tout autre gouvernement, sur où, quand ou comment ce gouvernement décide d'avoir recours à la force dans le cadre des opérations de maintien de la paix de l'ONU. (Le Monde, 1994)

(...) je suis en train de parcourir le net pour me renseigner sur si je pourrais faire avec un mac tous ce que je fais avec mon pc (Internet)

Notre premier objectif est de présenter, indépendamment d'un cadre théorique particulier, la distribution des I.I. introduites par la préposition sur. Nous décrivons la distribution de ces compléments dans la position prépositionnelle des verbes à deux compléments.

Nous examinons également la possibilité d'effacement de la préposition introduisant une I.I. En effet, parallèlement à une I.I., on peut souvent observer une complétive de la forme ce $Q u P$ introduite par une préposition. Dans ce cas, il arrive que la préposition, souvent de ou à, et le mot ce puissent ne pas apparaître, laissant en surface une construction en apparence directe. Nous allons observer cette possibilité d'effacement de la préposition dans le cas des I.I.

\section{Quelques points de repère}

\subsection{Grammaires de référence}

Wagner \& Pinchon (1962: 572) mentionne la possibilité d'occurrence des interrogatives indirectes comme complément indirect qu'ils nomment "complément déterminatif". Ils citent l'exemple suivant de Voltaire :

L'impitoyable bailli, qui ne pouvait réprimer sa fureur de questionner, poussa enfin la curiosité jusqu'à s'informer de quelle religion était le Huron (Voltaire)

D'après Grevisse \& Goosse (1993: 1682-1683), la proposition d'interrogation indirecte peut correspondre à un complément nominal prépositionnel et cette préposition est parfois présente dans l'interrogation indirecte partielle. Cette compatibilité est régulière avec ce qui/ce que. Mais les exemples cités sont tous construits avec des verbes qui introduisent un complément prépositionnel en de, sauf un exemple avec un complément en sur (§1104, Remarques 2) : 
Si donc l'on m'interroge ; si l'on s'inquiète [...] DE ce que j'ai "voulu dire" dans tel poème, je réponds que je n'ai pas voulu dire, mais voulu faire (Valéry)

On m'interrogea [...], sur la politique, SUR ce qu'on pensait dans le public des affaires du Tonkin (Maupassant)

Les auteurs indiquent également que d'autres interrogatifs peuvent se combiner avec une préposition, mais dans une langue "moins soignée", "reflet de l'oral familier".

Cette description de Grevisse \& Goosse n'explicite pas les conditions d'apparition facultative ou obligatoire d'une préposition qui introduit une interrogative indirecte. Nous présentons dans ce qui suit quelques faits distributionnels qui montrent que l'apparition ou non de la préposition devant une I.I. dépende de plusieurs paramètres.

\section{2 [pc z.] et [Prép z.]}

Du point de vue de sa constitution intérieure et de sa distribution, une I.I. appartient, comme la complétive, à la catégorie des phrases subordonnées. Diverses études ont montré, cependant, que les I.I. et les complétives ne peuvent pas être traitées de la même façon ${ }^{2}$. Il n'en reste pas moins que des propriétés communes existent. Il arrive qu'on puisse observer, parallèlement à une complétive prépositionnelle Prép ce $Q u P$, l'occurrence d'une complétive directe $Q u P$ :

Luc a informé Léa de ce que Max est parti = Luc a informé Léa que Max est parti

Luc a consenti à ce que Léa vienne = Luc a consenti que Léa vienne

M. Gross $(1968,1975)$ a considéré ce parallélisme comme une transformation harrissienne et l'a appelé [pc z.]. D'après ses analyses, les complétives directes prennent cette forme suite à la réduction $(z=$ zéro $)$ de la préposition $(p)$ et du mot ce $(c)$ : pour montrer l'origine commune, la $Q u P$ directe se comporte comme son homologue prépositionnelle, en ce qui concerne la pronominalisation :

Luc a informé Léa que Max est parti = Luc en a informé Léa

Luc a consenti que Léa vienne = Luc y a consenti

Ces phénomènes qui portent sur la complétive ont été interprétés en profondeur par Muller (2001, 2004), bien que son étude ne porte pas spécifiquement sur les I.I.

Gross (1975 : 66-67) note à propos de l'IIT qu'elle ne peut pas être précédée d'une préposition et cite les exemples :

Paul avertira Jean si Marie est arrivée ou non

? Paul renseignera Jean si Marie est arrivée ou non

Mais il note en même temps que, puisqu'on peut observer des formes "incorrectes" comme :

Paul (décidera + parlera) de s'il peut le faire ou non

la transformation de [pc z.] ou [Prép z.] ne suffira pas à régulariser la distribution de l'IIT dans une position prépositionnelle. M. Gross ne décrit pas l'IIP dans ce cas de figure, mais la paire de phrases cidessous montre clairement la nécessité d'élargir la description à cette catégorie de l'I.I. :

Pas une vendeuse n'est venue m'avertir de pourquoi je dois attendre (Internet)

(...) pour les avertir pour qui on va voter dans 1 an, faut les prévenir combien de mois à l'avance (Internet)

La transformation [Prép z.] régularise la distribution, quand on décrit pour chaque verbe à préposition 1) la possibilité d'apparition d'IIT et d'IIP dans cette position et 2) la possibilité de trouver des IIP et IIT directes là où l'on s'attend à des IIP et IIT prépositionnelles. 
Dans ce qui suit, nous prenons comme objet d'examen quelques verbes à complément en sur et vérifions d'abord la distribution d'IIT et d'IIP. Nous comparons ensuite les phrases avec sur et celles sans sur.

\section{Les verbes à deux compléments}

Nous présentons la distribution des interrogatives indirectes comme complément prépositionnel en sur. Dans cette section, nous examinons des verbes à deux compléments, dont un objet direct :

$$
N_{0} V N_{l} \text { sur (si P ou si } P+Q u-P_{-Q u} \text { ) }
$$

Dans la description des verbes français du lexique-grammaire ${ }^{3}$, il n'existe pas de classe spécifique pour les verbes qui prennent un complément prépositionnel en sur. Ils sont répartis dans plusieurs classes différentes selon la nature morpho-syntaxique des arguments. Par exemple, on peut trouver plusieurs verbes que nous examinons dans cette étude dans la classe 16 définie par la construction suivante ${ }^{4}$ :

$$
N_{0} \text { V Prép } N_{1} \text { Prép } N_{2}
$$

Cette classe regroupe théoriquement les verbes à deux complétives post-verbales avec prépositions. Par exemple, les phrases suivantes sont cataloguées dans la classe :

\section{$N_{0}$ V (Prép ce) Qu P Prép ce Qu P \\ Luc s'aperçoit (de ce) que Léa vient de passer par là, à ce qu'il y a un léger parfum d'agrumes dans l'air}

\section{Luc a conclu que Léa l'a vraiment quitté de ce que son armoire était totalement vide}

Mais y sont également inclus les verbes dont le premier complément est construit directement (c'est-àdire : Prép = zéro) à la base.

Dans ce groupe de verbes, du point de vue de la distribution des interrogatives indirectes, nous pouvons distinguer deux classes de verbes, selon leur sémantisme : d'un côté, des verbes dont le sens implique une attitude vis-à-vis du savoir, de l'autre des verbes qui n'ont pas ce trait ${ }^{6}$.

Dans ce qui suit, nous allons vérifier pour chaque verbe les possibilités de sélectionner, comme complément prépositionnel, 1) une interrogative indirecte totale (IIT), 2) une interrogative indirecte partielle (IIP) de forme ce qui/ce que, 3) une IIP d'autres formes que ce qui/ce que et 4) une IIP qui contient une préposition dans son terme $Q u$ -

Avant la description détaillée, une remarque s'impose : dans tous les cas, l'acceptabilité de l'I.I. introduite par une préposition est strictement jugée au niveau oral, style familier. Effectivement, dans les écrits, il y a très peu d'occurrences de ces expressions, suite à des contraintes dont la nature reste obscure. Nous avons considéré, dans cette étude encore préliminaire, que, si ces expressions sont jugées "acceptables", elles reflètent un certain système syntaxique que possèdent les locuteurs français ${ }^{7}$.

\subsection{Verbes plus ou moins interrogatifs}

Il s'agit ici des verbes interroger, questionner et renseigner. Indépendamment de l'intuition sémantique, il $\mathrm{y}$ a un critère syntaxique qui distingue le complément prépositionnel de cette classes de verbes de l'autre classe que nous allons examiner ultérieurement : avec ceux-ci, il n'est pas si évident que le complément prépositionnel soit un argument du verbe. Syntaxiquement, on peut observer son détachement en tête de phrase et, en général, la préposition sur est interchangeable sans modification de sens avec la locution prépositionnelle à propos de :

\section{La commission a interrogé Léa (sur + à propos de) son comportement des derniers jours}

$=($ Sur + à propos de) son comportement des derniers jours, la commission a interrogé Léa 
Mais le test du remplacement du syntagme verbal par le faire n'exclut pas l'intégration du complément en sur :

?* Luc a interrogé Marie sur l'endroit de la réunion et Paul l'a fait aussi sur l'heure de la réunion

Luc a interrogé Marie sur l'endroit de la réunion et Paul l'a fait aussi

Dans cette étude, nous avons simplement considéré que le complément de "thème" ou de "sujet" que représenterait le complément en sur fait partie de la liste des arguments de ces verbes, sans entrer dans le détail de cette question.

\subsubsection{Complétive en ce que}

Les verbes que nous examinons sont classés dans la table 16 donc ils sont censés prendre une (ou deux) complétives. Les verbes interroger, questionner et renseigner sont définis avec la propriété positive pour sur ce que Pind. Bien que sur le fait que $P$ soit acceptable avec ces verbes, les phrases du schéma $N_{0} V N_{I}$ sur ce que $P$ sont difficilement acceptables :

?* La commission a (interrogé + questionné) Léa sur ce qu'il y avait chaque mois un versement de 3000 euros sur son compte bancaire pendant plusieurs années

?* Luc a renseigné Léa sur ce que le patron devait quitter la maison

La commission a (interrogé + questionné) Léa sur le fait qu'il y avait chaque mois un versement de 3000 euros sur son compte bancaire pendant plusieurs années

Luc a renseigné Léa sur le fait que le patron devait quitter la maison

Cette distribution, ainsi que ce que nous allons observer ci-dessous, met clairement en évidence le fait qu'une interrogative indirecte a sa propre distribution, indépendamment de celle de la complétive ${ }^{8}$.

\subsubsection{IIT}

La préposition sur introduisant une IIT est difficile à omettre dans une construction transitive :

La commission a (interrogé + questionné) Léa $\left({ }^{*} E+s u r\right)$ si elle avait eu une relation extraconjugale ou pas

Luc a renseigné Léa $\left(?{ }^{*} E+\right.$ sur $)$ si son mari la trompait ou pas

Cette gradation d'acceptabilité ne change pas si les phrases sont mises à la voix passive :

Léa a été (interrogée + questionnée + renseignée) (par la commission) (?*E + sur) si elle avait eu une relation extraconjugale ou pas

Léa a été renseignée $\left({ }^{*} E+\right.$ sur) si son mari la trompait ou pas

Cependant, si le verbe est d'un emploi réflexif, la bizarrerie semble s'atténuer :

Léa s'est interrogée $(? E+$ sur) si elle aimait Luc vraiment ou pas

Léa s'est renseignée $(? E+$ sur) si son mari la trompait ou pas

\subsubsection{IIP avec ce quilce que}

La présence de la préposition est obligatoire si l'IIP est en ce qui/ce que :

La commission a (interrogé + questionné) Léa $\left({ }^{*} E+\right.$ sur) (ce qui avait provoqué le divorce + ce qu'elle reprochait le plus à son ex-mari)

Luc a renseigné Léa (*E + sur) (ce qui n'allait pas bien + ce qu'il comptait faire) 
Cet état de fait ne change pas si les phrases sont mises à la voix passive :

Léa a été (interrogée + questionnée) (par la commission) $\left(^{*} E+\right.$ sur) (ce qui avait provoqué le divorce + ce qu'elle reprochait le plus à son ex-mari)

Léa a été renseignée ( ${ }^{*} E+$ sur) (ce qui n'allait pas bien + ce que Luc comptait faire)

Contrairement à l'IIT, la préposition est obligatoire avec l'emploi réflexif du verbe :

Léa s'interroge $\left({ }^{*} E+\right.$ sur) (ce qui avait provoqué le divorce + ce qu'elle reprochait le plus à son ex-mari)

Léa s'est renseignée (*E + sur) (ce qui n'allait pas bien + ce que Luc comptait faire)

\subsubsection{IIP d'autres types}

La présence de la préposition est toujours obligatoire avec les IIP autres que celles en ce qui/ce que. Apparemment, il ne semble pas y avoir de restriction sur les éléments $Q u$ - :

La commission a (interrogé + questionné) Léa $(* E+$ sur) (qui avait organisé la réunion + pourquoi elle avait organisé la réunion)

Luc a renseigné Léa (*E + sur) (qui avait été invité à la soirée + pourquoi elle n'a pas été invitée)

Que ce soit avec le pronom interrogatif humain qui ou l'adverbe interrogatif pourquoi, l'IIP est introduite par la préposition sur, mais son omission ne donne pas une phrase acceptable. La même acceptabilité persiste avec les phrases passives. Mais avec l'emploi réflexif, l'acceptabilité de certains types de mots Qu-s'améliore :

Léa s'est (interrogée + renseignée) (*E + sur) qui Max avait invité à sa soirée

Léa s'est (interrogée + renseignée) (?E + sur) pourquoi Max ne l'avait pas invitée

De plus, l'acceptabilité est meilleure quand le verbe est à la première personne du singulier :

Je (m'interroge + vais me renseigner) (pourquoi + comment) Max a fait cette chose

\subsubsection{IIP prépositionnelle}

Si une IIP comporte un élément $Q u$ - dont la fonction interne est indirecte, deux prépositions apparaissent à la surface. Normalement, la préposition de l'élément $Q u$-n'interfère pas avec la sous-catégorisation du terme recteur ${ }^{9}$, comme le montre l'exemple suivant où le verbe transitif savoir accepte une IIP avec $Q u$ prépositionnel :

Luc ne sait pas à qui Léa a donné la clef de son bureau

Il est possible d'imaginer que la préposition du verbe qui introduit une IIP n'empêche pas l'apparition d'une préposition à la tête de celle-ci, probablement dans un noeud $C O M P^{10}$. Ça semble être le cas, et l'omission de la préposition du verbe recteur est même interdite, dans les exemples suivants :

(1) La commission a (interrogé + questionné) Léa $\left(^{*} E+\right.$ sur) de qui il s'agissait

Léa a été (interrogée + questionnée) $(* E+$ sur) de qui il s'agissait

sauf la phrase avec le verbe réflexif, comme c'était le cas dans les exemples précédents :

Léa s'interroge (?E + sur) de qui il s'agissait

Un cas de figure spécial à vérifier est celui d'une IIP prépositionnelle dont la préposition est identique à celle du verbe principal, en l'occurrence sur. Dans ce cas là, contrairement aux cas où la préposition de l'IIP est différente de sur, les phrases où les deux prépositions identiques se succèdent ne sont pas 
acceptables, tandis que les phrases où manque l'une des deux prépositions sont relativement meilleures que celles de (1):

* La commission a (interrogé + questionné) Léa sur sur quoi portait la conversation

(2) ?* La commission a (interrogé + questionné) Léa sur quoi portait la conversation

Il y a une différence d'acceptabilité dans la construction réfléchie correspondante. La succession des deux prépositions n'est pas totalement exclue :

?*Léa s'interroge sur sur quoi portait la conversation entre Luc et Max

L'occurrence de la seule préposition sur est meilleure qu'en (2) :

? Léa s'interroge sur quoi portait la conversation entre Luc et Max

La raison de ces différences n'est pas évidente et pour les analyser entièrement, il faudrait faire intervenir des considérations phonétiques et prosodiques.

\subsection{Les verbes du type baser}

Le deuxième groupe de verbes, qui entrent dans une construction à double objet dont l'un est un complément prépositionnel en sur, est composé de baser, fonder et asseoire. Cette classe de verbe n'évoque pas directement la notion énociative de question ou d'interrogation. Syntaxiquement, contrairement à la classe précédente, le complément prépositionnel semble être un argument du verbe et un complément essentiel obligatoire. La préposition n'alterne pas avec à propos de.

\subsubsection{Complétive en ce que}

Contrairement aux verbes observés, les verbes de cette classe acceptent bien la complétive en ce que, parallèlement au syntagme nominal complexe le fait $Q u$ P. Mais l'effacement de la préposition sur et de ce ne sont pas possibles :

L'avocat a (basé + fondé + assis) sa plaidoirie sur (ce + le fait) que l'accusé se trouvait à $5000 \mathrm{~km}$ de l'endroit du crime

* L'avocat a (basé + fondé + assis) sa plaidoirie que l'accusé se trouvait à $5000 \mathrm{~km}$ de l'endroit du crime

La passivation et l'emploi du réflexif neutre montrent exactement la même configuration.

\subsubsection{IIT}

Cette classe de verbes accepte une IIT introduite par la préposition sur mais celle-ci est obligatoire, dans quelque type d'emploi verbal que ce soit :

Ce linguiste a (basé + fondé + assis) son argumentation $\left({ }^{*} E+\right.$ sur) si une capacité langagière est innée ou pas

L'argumentation de ce linguiste est (basée + fondée + assise) (*E + sur) si une capacité langagière est innée ou pas

$L^{\prime}$ argumentation de ce linguiste se (base + fonde) $\left({ }^{*} E+\right.$ sur) si une capacité langagière est innée ou pas

Comparée aux exemples réflexifs des verbes interroger et renseigner, l'inacceptabilité du dernier exemple est remarquable. 


\subsubsection{IIP avec ce quilce que}

La configuration des acceptabilités est similaire à celle qu'on trouve avec les IIT. En aucun cas, la préposition sur ne peut disparaitre de la surface :

L'avocat a (basé + fondé + assis) sa plaidoirie $\left({ }^{*} E+\right.$ sur) (ce qui avait réellement eu lieu + ce que l'accusé avait réellement fait)

La plaidoirie de l'avocat est (basée + fondée + assise) (*E + sur) (ce qui a réellement eu lieu + ce que l'accusé a réellement fait)

La plaidoirie de l'avocat se (base + fonde) $\left({ }^{*} E+\right.$ sur) (ce qui a réellement eu lieu + ce que l'accusé a réellement fait)

Avec ce type d'IIP, il est impossible d'omettre la préposition sur, ce qui était déjà le cas observé avec les verbes du type interroger.

\subsubsection{IIP d'autres types}

La préposition sur ne peut non plus disparaître dans d'autres cas comme :

L'avocat a (basé + fondé + assis) sa plaidoirie $\left({ }^{*} E+\right.$ sur) quand et où l'accusé avait été vu

La plaidoirie de l'avocat est (basée + fondée + assise) ( ${ }^{*} E+$ sur) quand et où l'accusé a été vu

La plaidoirie de l'avocat se (base + fonde) $\left({ }^{*} E+\right.$ sur) quand et où l'accusé a été vu

\subsubsection{IIP prépositionnelle}

La configuration est la même que dans tous les autres cas : la préposition sur est obligatoire dans tous les contextes :

L'avocat a (basé + fondé + assis) sa plaidoirie ( ${ }^{*} E+$ sur) pour qui l'accusé avait pu appeler la victime le jour du crime

La plaidoirie de l'avocat est (basée + fondée + assise) $\left({ }^{*} E+\right.$ sur) pour qui l'accusé a pu appeler la victime le jour du crime

La plaidoirie de l'avocat se (base + fonde) ( ${ }^{*} E+$ sur) pour qui l'accusé a pu appeler la victime le jour du crime

Si la préposition du terme $Q u$ - de l'IIP est sur, la co-occurrence de celle-ci avec la même préposition verbe est interdite :

* L'avocat a (basé + fondé + assis) sa plaidoirie sur sur quoi portait la conversation entre l'accusé et la victime

La seule configuration où on observe une acceptabilité, c'est dans les phrases avec un emploi pronominal du verbe :

* La plaidoirie de l'avocat se (base + fond) sur sur quoi portait la conversation entre l'accusé et la victime

Contrairement aux verbes s'interroger, les verbes pronominaux de cette classe refusent nettement la succession des deux prépositions. Cependant, par rapport à celles-ci, les phrases suivantes sonnent mieux, comme c'était le cas pour le verbe s'interroger :

? La plaidoirie de l'avocat se (base + fonde) sur quoi portait la conversation entre l'accusé et la victime 


\subsection{Remarques}

Voici le tableau récapitulatif des distributions :

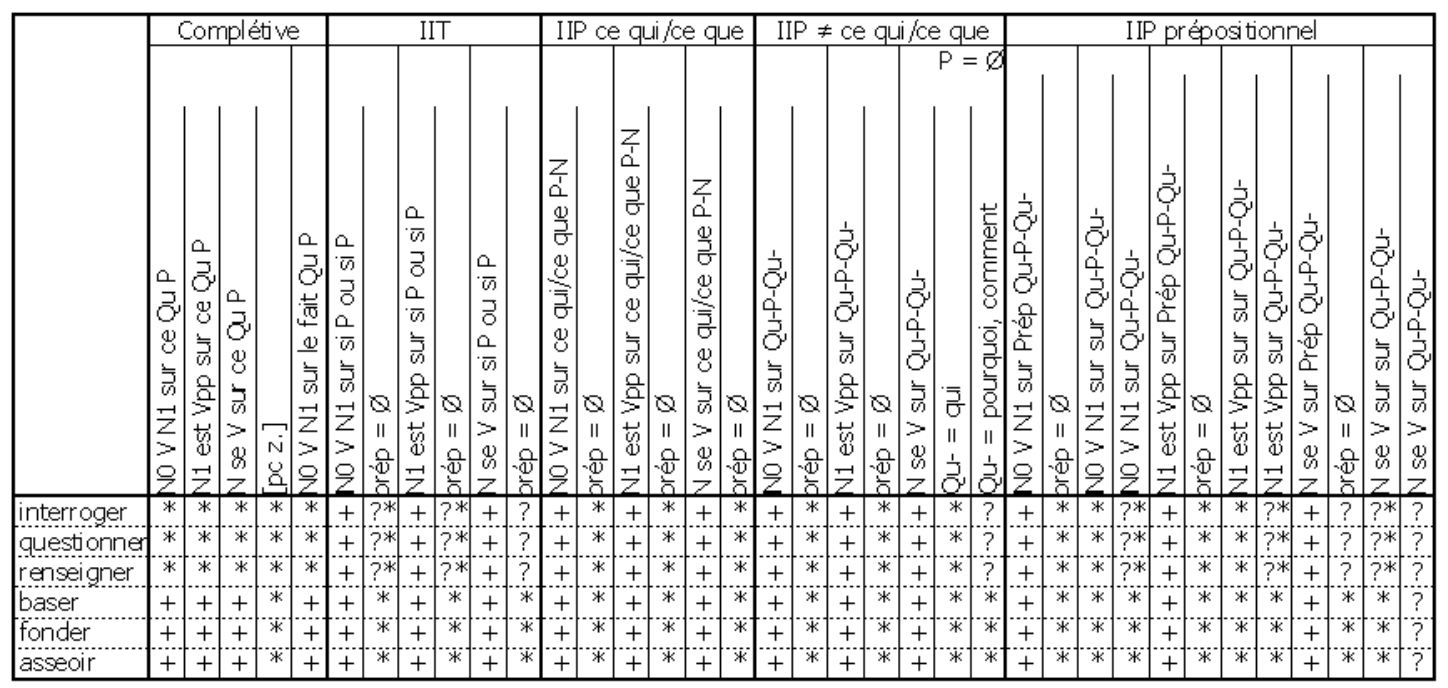

Tableau 1

Dans ce tableau, un signe qui apparaît à l'intersection d'une ligne et d'une colonne montre l'acceptabilité du verbe de la ligne pour la construction donnée dans la colonne. Dans l'ordre décroissant d'acceptabilité, on peut les énumérer de la façon suivante : $+, ?, ?^{*}, *$.

\subsubsection{Remarques générales}

Les verbes de la première classe (3.1.) n'acceptent pas de complétive, tandis que ceux de la deuxième classe (3.2.), oui. De ce point de vue-là, les verbes de la $1^{\text {ère }}$ classe sont comme le verbe se demander, qui n'accepte que les I.I., à l'exclusion de la complétive.

À part les complétives, la distinction intuitive des deux classes de verbes est corroborée par le fait que le groupe représenté par le verbe baser n'accepte pas du tout l'omission de la préposition sur qui introduit une I.I., sauf dans le cas où ces verbes s'emploient dans leur forme pronominale et que l'IIP est prépositionnelle en sur.

En ce qui concerne l'omission de la préposition, l'observation des comportements de la classe de verbes représentée par interroger montre que l'omission avec l'IIT est généralement plus facile que celle avec l'IIP et que l'omission de la préposition est plus facile quand le verbe est à la forme pronominale.

L'omission de la préposition avec la série IIP est très contrainte et la possibilité semble dépendre de la nature des éléments $Q u$ - et de la construction verbale : en général, seules les formes pronominales acceptent relativement facilement l'omission de la préposition sur, si l'élément $Q u$ - appartient à la classe des éléments adverbiaux ou prépositionnels, au détriment des éléments $Q u$ - nominaux argumentaux comme qui, ce qui, ce que.

\subsection{2}

Il n'est pas évident d'évaluer pourquoi l'omission de la préposition sur est difficile avec des verbes transitifs comme ceux que nous avons examinés, surtout quand ils sont suivis des IIP. Le fait que les verbes pronominaux s'interroger et se renseigner acceptent relativement facilement les I.I. sans qu'il y ait la préposition du verbe fait penser à un facteur syntagmatique : si l'I.I. est plus proche d'un $V$, voire directement après le $V$, l'acceptabilité augmente par rapport au cas où elle se trouve derrière un autre 
syntagme nominal. Mais cette hypothèse n'expliquerait pas pourquoi l'IIT dans une forme active transitive sans la préposition du verbe est mieux acceptée qu'une IIP dans la même configuration. Cette hypothèse ne rend pas plus compréhensible le fait que le deuxième groupe de verbes n'accepte en aucun cas l'effacement de la préposition.

\subsection{3}

Pour ce qui concerne le dernier point, bien que son apport ne soit pas clair pour l'instant, il est intéressant de revenir sur le statut de la préposition sur. Cette préposition qui se construit avec le premier groupe de verbes possède un statut de prédicat, si l'on tient compte des exemples nominalisés :

\section{L'interrogation de Léa par Max (est + porte) sur comment elle a réussi à séduire le président \\ Le (renseignement + question) (est + porte) sur qui a pu commettre un meurtre}

La décomposition de ces exemples donnera les schémas suivants :

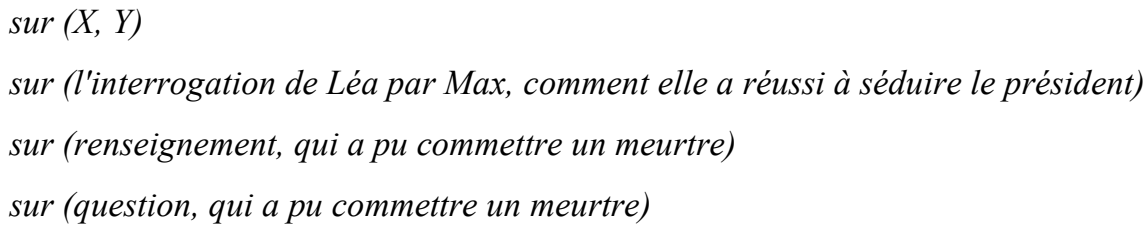

Dans les phrases verbales, la préposition sur semble assumer un rôle prédicatif similaire.

D'un autre côté, avec le deuxième groupe de verbes, la même préposition ne pourrait pas être analysable de la même façon :

$$
\begin{aligned}
& L E \text { (base + fondement) de la plaidoirie }\left(d e+{ }^{*} \text { par) l'avocat est }\left(E+{ }^{*} \text { sur }\right)\right. \text { comment l'accusé a } \\
& \text { pu se trouver au même endroit que la victime le jour du meurtre }
\end{aligned}
$$

Le deuxième complément phrastique du verbe sert de complément d'apposition au verbe nominalisé et la préposition sur n'a aucun rôle à jouer dans cette relation. C'est un simple marqueur de fonction dans une construction verbale.

Mais ces considérations nous mèneraient à une conclusion contraire à nos observations : si la préposition sur joue un rôle prédicatif, elle ne pourrait pas être omise dans une construction verbale de la première classe de verbes et si elle ne joue qu'un rôle de marqueur de fonction inhérent au verbe, elle pourrait être omise dans une construction verbale avec la deuxième classe de verbes. Or, bien évidemment, c'est le contraire que nous avons observé. Nous n'avons pas de réponse à cette question pour l'instant.

\section{Conclusion}

Nous avons décrit dans cette étude la distribution des interrogatives indirectes totales et partielles, dans les cas où elles sont des compléments prépositionnels en sur de verbes à deux compléments. D'après cet examen, l'I.I. peut apparaître dans une position syntaxique où une complétive est exclue et où l'omission de la préposition dépend de plusieurs facteurs : la nature du verbe principal (verbes du type interroger vs. verbes du type baser), la nature des éléments $Q u$ - (IIT vs. IIP, $Q u$ - nominaux argumentaux vs. $Q u$ adverbiaux), la nature de la préposition de l'élément $Q u-$.

Comme perspective, nous avons l'intention d'élargir l'étude à d'autres types de verbes, notamment les verbes intransitifs qui se construisent avec un complément en sur. L'acceptabilité des I.I. étant meilleure avec les verbes pronominaux, on peut faire l'hypothèse que l'I.I. s'accepte mieux avec les verbes intransitifs et que la préposition peut être omise plus facilement. 


\section{Références bibliographiques}

Boons, J.-P., A. Guillet \& C. Leclère. (1976a). La structure des phrases simples en français : constructions intransitives. Genève : Droz

Boons, J.-P., A. Guillet \& C. Leclère. (1976b). La structure des phrases simples en français : classes de constructions transitives. Rapport de recherches du L.A.D.L. $\mathrm{n}^{\circ} 6$ : Paris.

Borillo, A. LLL

Grevisse, M. (1993). Le Bon Usage (13 ${ }^{\text {ème }}$ édition, revue et refondue par Goosse, A.). Paris/Louvain-la-Neuve : Duculot.

Gross, M. (1975). Méthodes en syntaxe : régimes des constructions complétives. Paris : Hermann.

Guillet, A. \& C. Leclère. (1992). La structure des phrases simples en français : constructions transitives locatives. Genève : Paris.

Muller, C. (1989). Sur la syntaxe et la sémantique des relatives indépendantes et des interrogatives indirectes partielles. Revue Romane, 24:1, pp. 13-48.

Muller, C. (1996). La subordination en français : le schème corrélatif. Paris : Armand Colin/Masson.

Muller, C. (2000). Prépositions et subordination en français. Scolia, 15, pp. 87-106.

Muller, C. (2001). Sémantique de la subordination. In Rousseau, A. (éd.), La sémantique des relations, Lille : Publications de l'université Charles-de-Gaulle - Lille 3, pp. 163-177.

Muller, C. (2004). À propos de [pc z.]. In Leclère, C. et al. (éd.), Syntax, Lexis \& Lexicon-Grammar, papers in honor of Maurice Gross, Amsterdam/Philadelphia : John Benjamins Publishing Co, pp. 439-453.

Wagner, R.L. \& J. Pinchon (1962). Grammaire du français classique et moderne. Paris : Hachette.

\footnotetext{
${ }^{1}$ L'exemple est ambiguë : qui l'écoute peut être interprété comme une relative indépendante. Mais cela n'empêche que cette position peut être occupée par une série d'I.I.P : Il dépend de quand, où, comment on l'écoute.

${ }^{2}$ Notamment, les études sur la subordination de Muller (1996).

${ }^{3}$ Voir Gross (1975), Boons, Guillet \& Leclère (1976a, b) et Guillet \& Leclère (1992).

${ }^{4}$ Les références aux classes du lexique-grammaire concernent un état actuel des tables, non publié. Par exemple, la classe 16 de Gross (1975) ne comportent pas de verbes que nous examinons dans cette section.

${ }^{5}$ Muller (2004) parle, à juste titre, de la possibilité de ne pas tenir compte de la complétive à ce $Q u$ P pour décrire la structure de phrase du verbe s'apercevoir. Ce verbe doit figurer dans la classe 8 comme Muller (2004) l'indique.

${ }^{6}$ La sélection d'une interrogative indirecte par un prédicat n'est pas dépendante du sens interrogatif du verbe. Sur ce point, voir Muller (1996, 2001).

${ }^{7}$ Indépendamment de notre intuition, nous avons systématiquement soumis les phrases d'exemples à l'examen d'un linguiste francophone d'origine. Les jugements que nous donnons aux exemples sont donc contrôlés par deux personnes.

${ }^{8}$ Muller (1996 : §3.2.1., 202-204) fait état de l'insuffisance d'identifier la distribution des interrogatives indirectes avec celle de la complétive.

${ }^{9}$ Muller (1996 : §3.1.3., 197).

${ }^{10}$ Voir pour ce point, Muller (1996).
} 